\title{
The promise of resonant inelastic X-ray scattering for modern Kondo physics
}

\author{
J. N. Hancock ${ }^{1, *}$ and I. Jarrige ${ }^{2}$ \\ ${ }^{1}$ Department of Physics and Institute for Materials Science, \\ University of Connecticut, Storrs, Connecticut, 06269 USA \\ ${ }^{2}$ National Synchrotron Light Source II, Brookhaven National Laboratory, Upton, NY 11973-5000, USA
}

\begin{abstract}
Developments in synchrotron accelerator and end station capabilities have brought new techniques to bear on complex matter. The recently-enabled technique of resonant inelastic X-ray scattering (RIXS) has emerged as a serious contender of inelastic neutron scattering as a probe of magnetic structure in correlated electronic materials, but can also reveal perspectives on electronic structural not attainable by optical conductivity or angle-resolved photoemission. Here we introduce the basics of this powerful technique and discuss the prospects to turn this probe onto the charge excitations in Kondo lattice systems.
\end{abstract}

PACS numbers: 71.20.Eh, 78.70.Ck, 72.15.Qm, 75.20.Hr

The study of the impurity Kondo problem in condensed matter has brought about many changes in physics, including the innovation of renormalization group formalism and which nucleated many notable contributions to theory of many body systems ${ }^{1}$. When the Kondo Hamiltonian is generalized once to include fluctuations of the valence state, and again to a periodic array of 'impurity' sites, one gets the periodic Anderson model (PAM), which contains a rich variety of electronic phases. Much of the physics of the heavy fermion class of materials is believed to be described by this electronic Hamiltonian, but many mysteries remain regarding the connection between theory and experiment, highlighting the importance of the development of new tools to probe complex correlated matter. Below, we contextualize the emerging technique of resonant inelastic X-ray scattering (RIXS) against related mainstay techniques and demonstrate its use to address the electronic structure of a particular material $\left(\mathrm{YbInCu}_{4}\right)$ in the physics of strongly correlated electron systems.

\section{RIXS AMONG OTHER X-RAY TECHNIQUES}

Core electrons are high energy bound electrons with filled-shell electronic configuration and wavefunctions which are extremely localized around the nucleus. For the pursuit of understanding materials behavior, these highly atomic electronic states are considered unimportant degrees of freedom because their excitations occur at a very high energy scale $(>10 \mathrm{eV})$ compared to even the largest scales of valence electron excitations. X-ray interaction with materials can excite the deeply bound core electrons and promote them to unoccupied states. Across many scientific disciplines, $\mathrm{X}$-ray spectroscopy is a mainstay of experimental research and characterization.

$\mathrm{X}$-ray absorption (XAS) is a very important scientific tool, which can be regarded on many different levels. Often, it is used to simply provide a chemical fingerprint permitting estimates of stoichiometry, while in more advanced treatments, detailed analysis of the near-edge structure can be used to classify the valence states of particular atomic species in nonconducting materials ${ }^{2,3}$. Prominent examples of spin-off variants of direct XAS are extended X-ray absorption fine structure (EXAFS) ${ }^{4,5}$, wherein the echoes of photo-excited electrons can be read from spectra to reconstruct the local environments of the resonant species, and X-ray magnetic linear/circular dichroism (XMLD/XMCD), wherein one senses the spin imbalance on a particular site through absorption of linear or chirally polarized photons ${ }^{6}$. Important work in the 1980s and $1990 \mathrm{~s}^{7,8}$ showed that this information was related in a simple way to the species-specific expectation value of orbital and spin magnetization components in the ground state, a development which catalyzed X-ray dichroisms rise to prominence as a materials characterization technique. Each of these probes are useful in their own right for the study of local structure and magnetic states in condensed matter.

When a core hole is photo-generated in a solid, this highly excited state awaits many possible fates that can be categorized into decay channels which eventually yield either a photon or a free electron (see Figure 1). The most versatile and common-practice method for collecting XAS data is through total fluorescence yield, where all photons emitted from the sample are collected without any special effort to discriminate their energies, polarization, or momenta. There are known conditions where direct absorption and total fluorescence yield give different results? ${ }^{\text {? }}$.

If the scattered photons are collected more selectively, using variable detection angle to segregate them by momentum, one has a powerful probe of electronic structure in real space with resonant elastic X-ray scattering $(\mathrm{REXS})^{9,10}$, also known as resonant diffraction. REXS is a fingerprint of static structures in matter complementary to neutron and nonresonant X-ray diffraction, which probes most directly magnetic and nuclear order. Because the REXS probe involves the highly atomic core levels, projections of atomic character of valence electron spin and orbital character are measured, giving a unique perspective in comparison to the more traditional techniques of neutron diffraction and nonresonant (Thomson) $\mathrm{X}$-ray scattering. 


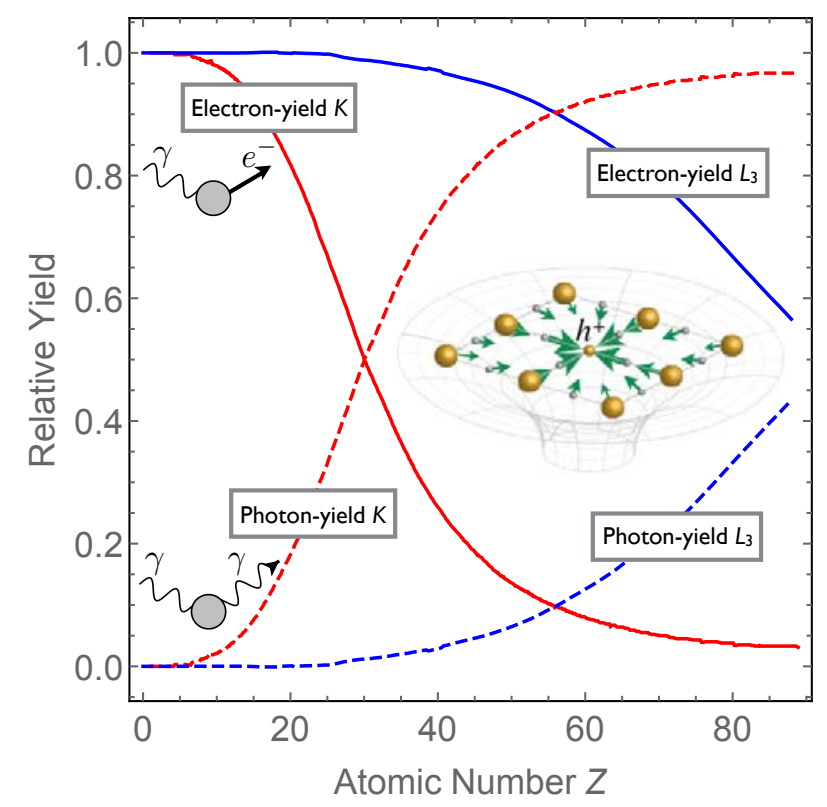

FIG. 1. Typical relative electron and photon yields resulting from X-ray excitation and for hole creation as a function of atomic number $Z$. For $Z>30$, which describes most rareearth and $5 d$ electron systems, photon yield is significant. Inset: Schematic showing the effects of core-hole creation on nearby valence electrons in a high- $\mathrm{T}_{c}$ superconductor structure.

If one discriminates the outgoing photons by energy as well as momentum, we have the developing technique of resonant inelastic X-ray scattering (RIXS). Differences in efficiency of this process for different kinematically distinct photon states carries direct information of the fundamental excitations of the material under study and is sensitive the fluctuations of charge and spin. The information carried by the photon field is very rich, and the ability to control and discriminate the photon degrees of freedom and prepare the state of the material in a wellcontrolled way is crucial to maximizing the return on the worlds RIXS investment.

\section{TYPES OF RIXS PROCESSES}

RIXS differs from XAS in that it discriminates the emitted photons in the core-hole decay process by energy and often momentum and polarization. As with resonant Raman spectroscopy, one is then concerned with the ground state of physical interest, an intermediate state (which contains a core-hole at an X-ray edge), and a final state. RIXS can further be subdivided into the categories of high energy transfer (HET-RIXS), wherein the final state also contains a core hole, and low energy transfer (LET-RIXS), wherein the final state again involves the lower-energy $(<10 \mathrm{eV})$ scales most relevant to material behavior.
HET-RIXS is often collected in a momentumintegrated fashion, as the process, highly atomic in nature, is not expected to have a strong momentum dependence. The kinds of information that one gleans regards the orbital occupancies of specific elements, and gives particularly strong contrast in systems which feature valence instabilities ${ }^{11-14}$. The experimental requirements of this form of spectroscopy are not highly demanding, due to the high signal level, muted momentum dependence, and intrinsic broadening of the core-hole-containing final states.

LET-RIXS on the other hand ultimately generates lowenergy excitations which are contained in the effective Hamiltonian relevant to the macroscopic low-energy behavior of physically interest. Far-reaching physical concepts arise from materials models in the spirit of the t-J, Hubbard, Holstein, periodic Anderson, and other minimal many-body Hamiltonians. As such, momentum dependence in LET-RIXS is not only expected, but constitutes a direct measurement of the dispersion of electronic and other collective excitations in the solid. During the recent period of rapid development, perspective on the character and dispersion of ligand orbital ${ }^{15-17}$, crystal field $^{18,19}$, orbiton ${ }^{20}$, magnon ${ }^{21-24}$, bimagnon ${ }^{24-26}$, paramagnon $^{27}$, polaron $^{28}$, Mott-gap ${ }^{16,29}$, and even spin2 triplon excitations ${ }^{30}$ has been delivered by LET-RIXS. In cases, coupling between these degrees of freedom have been revealed. Because of the sensitivity to the unique combination of atomic species, valence state, orbital character, momentum, and energy, the information brought by RIXS probes is precisely what is needed to bridge the gap between atomic physics and the collective behavior of materials. As a burgeoning spectroscopic probe, the full expanse of the information one can gain has yet to be realized, but its value to the science community has become indisputable in recent years following provision of fresh perspectives on pointed questions in quantum materials long left open by the traditional techniques of inelastic neutron scattering (INS) and angle-resolved photoemission spectroscopy (ARPES) $)^{11,27}$.

\section{RIXS AS A COMPLEMENT TO ARPES AND INS}

A particularly profound gateway result of RIXS is the experimental discovery that a single spin flip can be produced by purely electromagnetic means (LETRIXS). This effect is now well-understood ${ }^{31}$, and single magnons have been observed using RIXS spectroscopy in $5 d$ iridates $^{32}$ and $3 d$ transition metals ${ }^{21}$ with magnetic order. In cuprates, the magnetic signal has been tracked successfully to high doping levels, revealing that spin susceptibility exists in even strongly doped superconductors on the $300 \mathrm{meV}$ scale $^{27}$. An important theoretical element in the physics behind the scattering mechanism which makes these observations possible involves the extremely strong spin-orbit coupling of the $2 p$ core level 

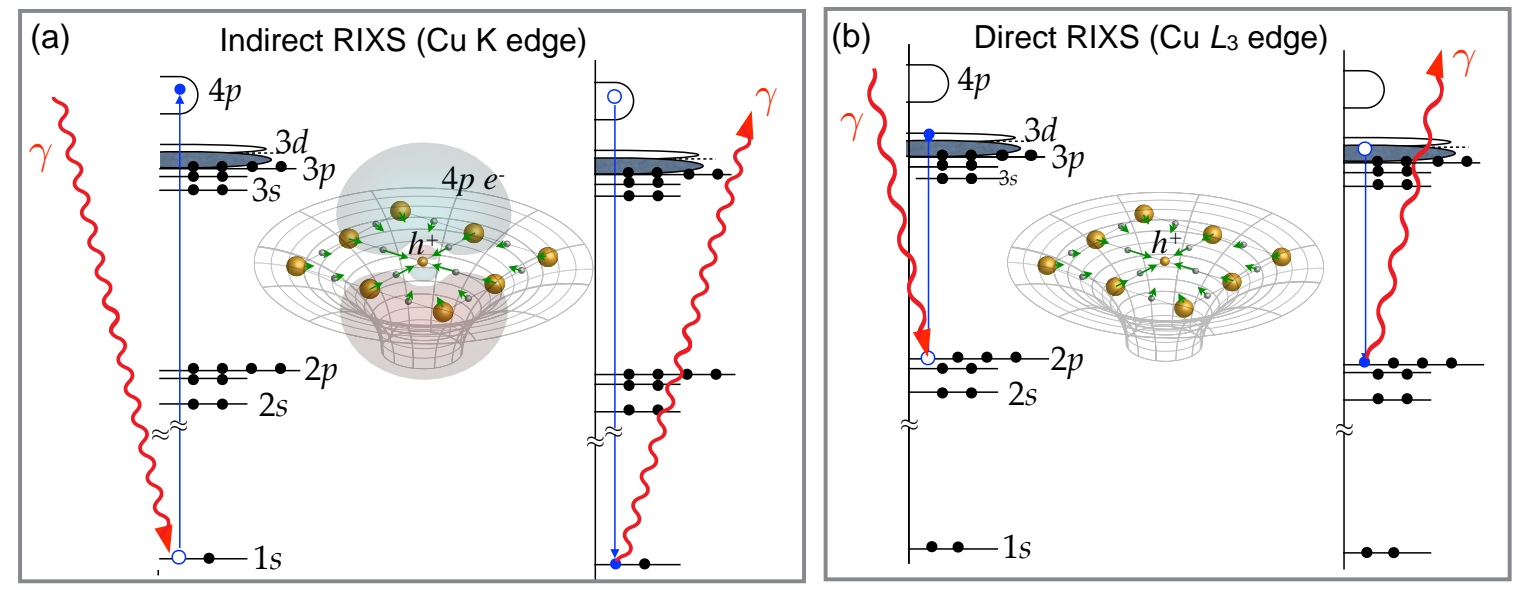

FIG. 2. Left: Schematic of the direct RIXS process, exemplified at the $\mathrm{Cu} K$ edge. A photon, shown as a red wavy line, excites a core electron $(2 p)$ into an unoccupied electronic state of $3 d$ character. The core hole potential and extra electron disturb the valence system so that when a recombination of a $d$ electron with the core hole occurs, the system is left in a low-lying state without core holes. Right: Indirect RIXS occurs when the photoelectron enters a state removed from the valence system. In this case, most of the disturbance of the valence system is a result of the core hole. Direct and Indirect RIXS shake up different excitations, and are therefore effectively distinct probes of matter. A given species usually has more than one experimentally accessible edge.

in a spin-polarized transition metal, which opens a photoelectron recombination pathway differing from the absorption pathway through the spin index ${ }^{31}$. The importance of this discovery cannot be overstated, as it provides a method of studying magnetic excitations with numerous advantages over neutron scattering. Indeed many groups across the world, whose activities were traditionally based in neutron scattering, have recently embarked on successful RIXS activities. RIXS has clear advantages over inelastic neutron scattering: (i) clear information on which orbitals contain spin, (ii) high spatial resolution, and avenues to magnon microscopy, (iii) high signal can probe small crystals, thin films, monolayers and neutron absorbers, (iv) higher pressures due to the smaller beam, and (v) nuclear byproducts are avoided entirely in the beam production.

Polarized X-ray processes promote bound electrons of well-defined parity and to unoccupied states. If the final state is unbound, the electron can be collected through angle-resolved photoemission spectroscopy (ARPES). In its essence, ARPES measures a projection of a manybody state with $N$ electrons into one with $N-1$. If the excited electron however recombines with the core hole and emits a photon, this is the process of RIXS. LET-RIXS ultimately makes photon-assisted transitions among lowenergy many electron states with $N$ total electrons which may be captured in a many-body model. Due to the separation of core and valence electronic states, simplifications occur and the intermediate states may have $N$ valence or $N+1$ valence electrons, depending on the edge of interest.

It is often the case that the photoelectron enters highly itinerant, unoccupied states far above threshold into states removed from low energy physics, so that the response of the valence states of interest can be described by an $N$-body virtual bound state with a core hole potential. This type of indirect RIXS transition, illustrated in Figure 2a, is realized at the transition metal $K$ edges where $4 p$ electrons $>10 \mathrm{eV}$ above the Fermi level are highly banded with each other and have low probability density everywhere. This process is effective at driving charge-transfer/Mott gap processes in transition metal oxides and is relatively symmetry-preserving to the valence states.

The other limit, so-called direct RIXS, illustrated in Figure 2b, involves the photoelectron entering directly the valence states, and the important $N$-body manifold which results in the material behavior, plus one where there are $N+1$ bodies and a core hole potential. This type of RIXS is exemplified at the transition metal $L$ edge, where the valence states take up a $d$ electron in the intermediate state. One sees then that the perspective that RIXS brings to electron spectroscopy probes the $N$-electron states of the system, but can give information about the $N$ or $N+1$ electron systems as well. It is therefore fully complementary to photoemission as a probe of many body physics of complex materials. The distinction between indirect RIXS, direct RIXS, and other spectroscopic probes is shown in Figure 3.

There are some clear advantages of RIXS over ARPES. In finite magnetic fields, for example, a kinematic conundrum renders all electron-based spectroscopies (EELS, ARPES) useless, and UHV conditions are not required for hard X-ray experiments. Briefly, RIXS surmounts several limitation of electron-based spectroscopies, because it is: (i) bulk sensitive, (ii) compatible with single 
crystals and films, (iii) inherently valence and symmetry selective through resonance tuning, (iv) high magnetic field compatible, and (v)high pressure compatible.

\section{RIXS IN KONDO MATERIALS}

A class of systems which appears to be clean, very tunable, and carry a rich variety of competing and coexisting phases of matter are the heavy fermion systems. They exhibit clear features of correlated electron systems like fully developed local magnetic moments at high temperature, but may also bear characteristics of strongly renormalized Fermi liquids at low temperature. Often, unusual scaling behavior is observed in close proximity to phase boundaries between ordered magnetic, metallic, and superconducting instabilities, and analogies between the physics of this class of materials and HTSC are likely to hold, emphasizing the importance of such pursuits. Central to the physics of this material class is the Kondo effect, wherein itinerant electrons, exchange coupled to local moments, screen the magnetic fluctuations in a many-body virtual bound state resonance ${ }^{33}$. The excitations of this many-body state can sometimes be described by a highly-renormalized Fermi liquid, which, when pushed to its extremity, often leads to a quantum phase transition and non-Fermi liquid physics. Renewed interest in this class of systems accompanies the topological revolution, as the spin-orbit interaction is very strong and important in rare earth intermetallic systems, and several members are candidates for nontrivial topological electronic behavior ${ }^{34,35}$.

The original Kondo hamiltonian is an integer-valence limit of the Anderson impurity model (AIM), which has the feature that the large energy scales of the kinetic energy of the itinerant electrons (bandwidth B) and hybridization $(\mathrm{V})$ of these electrons with localized moments, cooperate to form a region of high density of states near the Fermi surface which has significant localized character, the so-called Abrikosov-Suhl (aka Kondo) resonance $^{33}$. When the physics of the AIM are generalized to an array of impurities, one has the periodic Anderson model (PAM), which is believed to widely encompass the electronic physics of $f$-intermetallic heavy fermions in the Fermi liquid regime. Below a characteristic temperature $T^{*}$, which is of order the Kondo temperature $\left(T_{K}\right)$ opens in the density of electronic states resonance itself, with spectral weight piled high on either side. Analysis of the momentum-dependent spectral function shows that this large density of states has momentum structure, with a direct, or momentum-resolved gap set by a renormalized hybridization strength, which can be much larger than the temperature scales $T^{*}, T_{K}$ and ranges from the order 10 s to 100 s of $\mathrm{meV}$ for Ceand Yb-based heavy fermion systems in the Fermi liquid regime. Infrared (IR) spectroscopy can probe these excitations with high energy resolution, and a large body of work exists ${ }^{36-38}$, but the nature of the IR final states are near zero net momentum due to the kinematical constraints of the low-energy IR photons.

Generally, the hybridization matrix element carries structure of momentum inherited from the environment of the local moment, as overlap integrals are generically dependent on orbitals and directions as can be seen for example in a Slater-Koster analysis of electronic band structure. This directionality of hybridization is of high scientific interest, and a consequence that can have strong effects on the energetics and susceptibility, therefore tendencies toward instabilities, but are also the driving force behind the predicted topological states in some materials from this class. There exists a degree of particle-hole symmetry in the physics of the Kondo lattice, and the best expression of this in real materials is in comparisons of $\mathrm{Ce}$ and $\mathrm{Yb}$, which have nominally one electron, or hole, respectively in the f electronic shell. Photoemission experiments of course only remove electrons, and the hybridization gap and enhanced density of states region appears on the electronic side of the Fermi level for $\mathrm{Yb}$. Experiments have revealed indications of this structure by ARPES and scanning tunneling microscopy but results are limited by kinematics and surface peculiarities and either integrate the momentum structure or probe only particle removal spectra of the many-body system. While these results and research activity add to our understanding of the physics of the lattice Kondo problem, a momentum- and valence-sensitive probe of twoparticle excitations will provide new perspectives on the Kondo problem, particularly the momentum-dependent hybridization matrix element. This key parameter establishes a connection between localized and itinerant states, but is highly sensitive to the local environment of the $f$ electron and ligand states? .

Surprisingly, the heavy fermion class of systems is experimentally completely open to low energy-transfer RIXS, but can be used to answer pointed questions: What is the role of hybridization nodes in driving quantum phase transitions? What is the nature of the Kondo insulator pseudogap? Are there excitonic states in the pseudogap? Can the Kondo effect stabilize the spin liquid state? The developing ability to read the quantum numbers of the electromagnetic field around X-ray edge energies is likely to be an important complement to existing spectroscopies in answering these questions.

\section{RIXS IN YBINCU 4}

To make a real impact and advance our understanding of Kondo materials, the energy resolution must be sufficient to capture the low-energy physical processes relevant to material behavior. The current practical energy resolution for both hard and soft X-ray-based RIXS techniques is of order $50-100 \mathrm{meV}$, which is at at the higher end of the hybridization scale of $f$ electrons in inter metallic systems. The best case is found for $\mathrm{Yb}$, where the large electronic orbitals give strongest hy- 


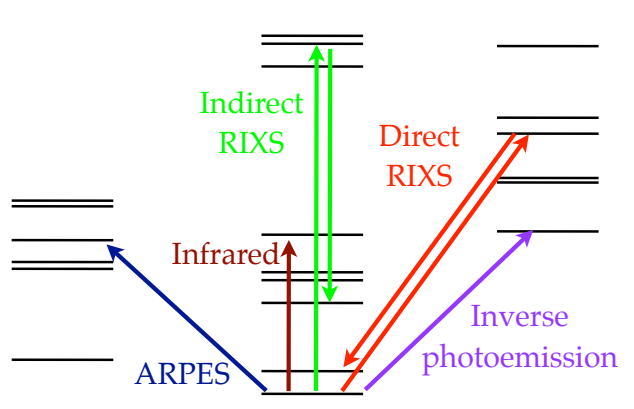

$\begin{array}{lll}N-1 & N & N+1\end{array}$

FIG. 3. Energy level diagram of a generic many-body system, showing how different probes of matter can excite materials among manifolds of different type. RIXS can couple the $N$ body system to manifolds of states which have $N$ or $N+1$ bodies, depending on whether RIXS is of the indirect or direct type, respectively.

bridization matrix elements, nearly $200-300 \mathrm{meV}$ in many cases. Although the resolution may be sufficient, there is a still a challenge to straightway identify feature associated with Kondo physics, mainly because of the presence of an elastic line near zero energy transfer, which can obscure inelastic features of interest. For this reason, we have chosen to first investigate $\mathrm{YbInCu}_{4}$, which has a valence transition associated with a a strong Kondoswitching behavior, permitting contrast on Kondo phenomena, while also permitting address of an important problem in strongly correlated electron systems.

The interest in $\mathrm{YbInCu}_{4}$ surrounds its temperaturedriven valence phase transition, where a hightemperature (HT) Curie paramagnetic phase which condenses into a low-temperature (LT) phase characterized by a Pauli paramagnetic spin susceptibility and reduced resistivity. Within either the LT or HT phase, many material properties can be described by a single Kondo temperature, with $T_{K}^{L T} \sim 300 K$ and $T_{K}^{H T} \sim 17 K^{39,40}$. Upon substitution of transition metals $\mathrm{YbXCu}_{4}(\mathrm{X}=\mathrm{Ag}, \mathrm{Mg}$, $\mathrm{Zn}, \ldots)$, the valence transition does not appear, and the behavior is more typical of members of the heavy fermion class of systems, which are continuously connected to the low- $T$ phase of the $\mathrm{X}=\mathrm{In}$ system $^{38,39,41}$. Calculations reveal the presence of a quasi-gap in the itinerant electronic band structure occurs in many of these compounds and for $\mathrm{X}=\mathrm{In}$, is closest to the Fermi level, a feature which was experimentally observed recently using RIXS at the $\mathrm{Yb}$ $L_{3}$ edge $^{42}$, along with concomitant changes in this quasigap and associated RIXS-induced electronic transitions. The observation of the interplay between textured itinerant state density and Kondo interactions is well beyond textbook treatments of Kondo and Anderson impurity physics $^{1}$, and is sufficient to influence phase stability in this material.

The temperature-driven phase transition in $\mathrm{YbInCu}_{4}$ permits investigation of materials behavior permitting
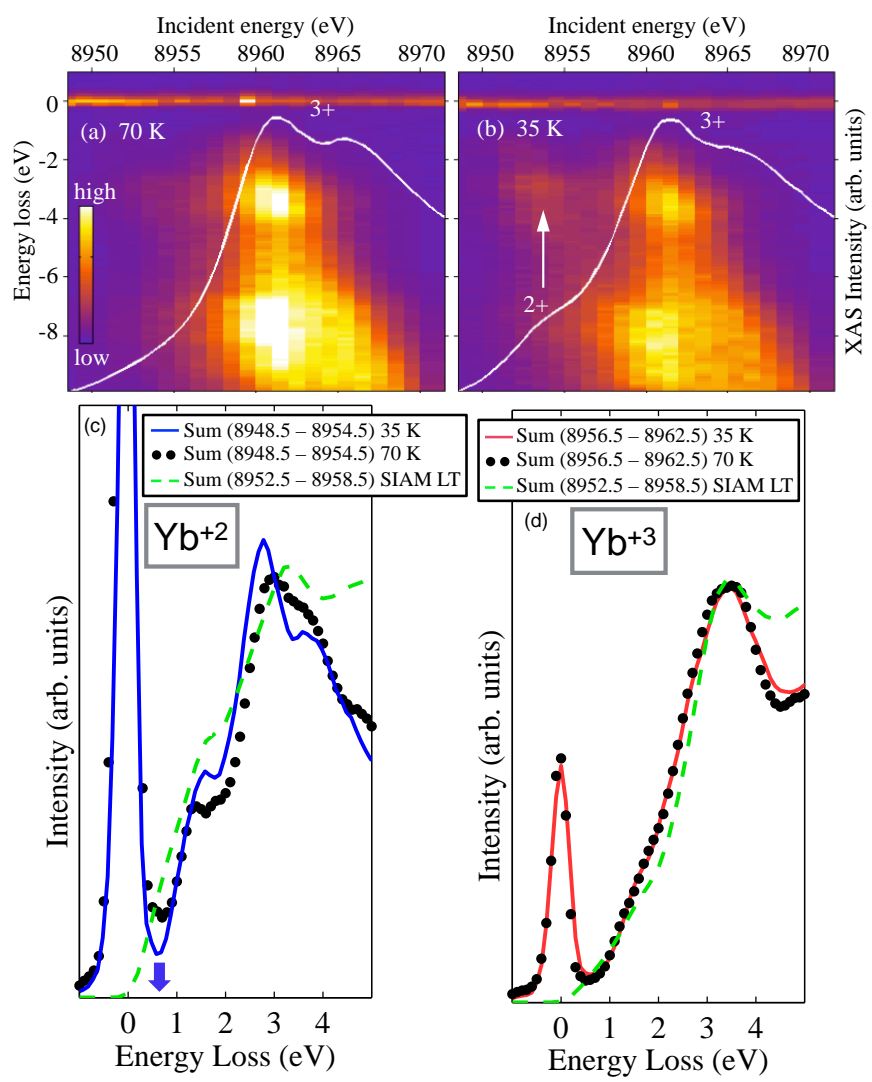

FIG. 4. RIXS spectral planes at at $35 \mathrm{~K}$ and $70 \mathrm{~K}$, with XAS spectra superimposed. From ${ }^{42}$

contrast between a virtually free moment system with one where mass enhancement and screening is a dominant aspect of the physics. Previous HET-RIXS studies in a scattering channel involved a final state with a core hole ${ }^{11}$ emphasized the bulk nature of the probe to resolve a long controversy in the photoemission data, clarified the nature of the excitation spectra through $T_{v}$. A more recent RIXS study probed the hard X-ray $\mathrm{Yb} L_{3}$ edge to excite electron-hole pair excitations among the $\mathrm{Yb} 5 d$ states. The present study involves a final state without a core hole, and so represents a penetrating probe of the low-energy excitations $(<1 \mathrm{eV})$ of this correlated electron system. The observation constitutes a demonstration of how the RIXS technique can be used to study the momentum-space texture of spin and charge excitations on the energy scales involved in the composite quasiparticles of heavy fermion and related systems.

Experiments were performed at the MERIX endstation of the XOR-IXS 30-ID beamline at the Advanced Photon Source ${ }^{43}$. $\mathrm{YbInCu}_{4}$ single crystals were aligned in a fourcircle diffractometer with an energy-dispersive analyzer arm based on diced Ge(008) spherical analyzer, which reflected scattered X-rays toward a strip detector placed on a 1-m Rowland spectrometer. The incident beam was monochromatized using a four-bounce scheme of asym- 
metrically cut $\mathrm{Si}(400)$ crystals. Taken together, we were able to achieve a resultant energy resolution of $200 \mathrm{meV}$ in the horizontal scattering geometry, with a scattering angle of $90^{\circ}$, horizontally polarized incident beam, and momentum transfer $Q=(5.5,3.5,3.5)$. The condition of $90^{\circ}$ is often used in RIXS experiments because the nonresonant Thomson elastic line is lowered significantly, permitting better access to lower-energy inelastic excitations.

Figs. 4a,b show XAS spectra as white curves for the high-temperature (HT, $70 \mathrm{~K}$ ) and low-temperature (LT, $35 \mathrm{~K}$ ) phases. The HT XAS curves shows structures at $8961 \mathrm{eV}$ and $8965 \mathrm{eV}$, representing excitation to absorption final states which contain a core-hole and having different electronic configuration among the valence electrons. These XAS final states are the intermediate states of the RIXS process, and have traditionally been used to distinguish valence configurations of materials. For example, the two peaks in the HT XAS spectrum represent a projection of the ground states onto the $4 f^{13}$ electron configuration $\left(\mathrm{Yb}^{3+}\right)$. At lower temperature of $35 \mathrm{~K}$, a shoulder emerges at a lower energy of $8953 \mathrm{eV}$, due to the emergence of $4 f^{14}$ character $\left(\mathrm{Yb}^{2+}\right)$ in the ground state, and effect of Kondo interactions below the valence transition. While this projection is useful, especially in high pulsed magnetic field measurements, the information gained is complicated by the presence of the core hole in the final state, and also is obfuscated by the finite lifetime of the core hole, which blurs any possible sharp features that would be observable directly in the XAS spectrum.

One possible recombination event following core-hole generation resulting from a $2 p \rightarrow 5 d$ photoexcitation is simply $5 d \rightarrow 2 p$ plus a valence system shakeup. The efficiency of this process is precisely the $L_{3}$ RIXS signal, shown as the RIXS spectral planes. These RIXS spectral planes allow one to separate at a glance those features which result from fluorescence, which would appear as diagonal streaks, from those which are true Raman excitations, which appear as localized regions or horizontal lines. Cooling below the transition results in some change of the features near 3 and $8 \mathrm{eV}$ energy loss, but the largest change occurs for the spectra collected at the $\mathrm{Yb}^{+2}$ state.

To simplify the interpretation of the RIXS data, we note that the incident energy axis breaks up into two resonances, those associated with the +2 state, and those associated with the +3 state. Averaging over each of these spectral regions allows us to isolate these two important RIXS spectra, shown in Figures 4c,d, respectively. Clearly, the +2 RIXS spectrum has the strongest changes in response to the phase transition, and important characteristics of this change are shifts of features at high energy, indicated by horizontal arrows, and the suppression of spectral weight at low energy when the Kondo screening effect turns on. This allows the first observation of electronic structure changes and in particular the quasi-gap below $1 \mathrm{eV}$, which has never been reported in the multiple infrared optical studies on this compound $^{36,38,41,44}$, but appears to be an important influence driving the phase transition.

In summary, we have contextualized RIXS in the backdrop of other X-ray spectroscopic techniques, highlighted the particular characteristics which distinguish it from conventional probes of ARPES and INS, and shown that its application to correlated materials which display aspects of Kondo phenomena can reveal information not otherwise attainable. The prospects for sustained contributions using RIXS in this area are strong, given the high interest in possible topological materials arising from spin orbit interaction.

We acknowledge valuable conversations with Akio Kotani. Work at the University of Connecticut is supported in part by National Science Foundation award DMR-1506825.
* jason.hancock@uconn.edu

1 K. Wilson, Reviews of Modern Physics 47, 773 (1975).

2 J. Stöhr, Book, Vol. 25 (1992) pp. xv, 403.

3 F. de Groot and A. Kotani, Advances in condensed matter science, v 6 (2008) pp. xx, 490 p.

${ }^{4}$ F. W. Lytle, J. Synchrotron Rad. 6, 123 (1999).

${ }^{5}$ P. a. Lee, P. H. Citrin, P. Eisenberger, and B. M. Kincaid, Reviews of Modern Physics 53, 769 (1981).

${ }^{6}$ S. W Lovesey and E. Balcar, "Quantum theory of natural circular, magneto-chiral and non-reciprocal linear dichroism," (2010).

7 J. Goedkoop, B. Thole, G. van der Laan, G. Sawatzky, F. de Groot, and J. Fuggle, Physical Review B 37, 2086 (1988).

8 B. Thole, P. Carra, F. Sette, and G. van der Laan, Physical Review Letters 68, 1943 (1992).

9 P. Abbamonte, L. Venema, A. Rusydi, G. A. Sawatzky, G. Logvenov, and I. Bozovic, Science (New York, N.Y.)
297, 581 (2002), arXiv:0208289 [cond-mat].

10 V. Scagnoli, U. Staub, Y. Bodenthin, R. A. de Souza, M. García-Fernández, M. Garganourakis, A. T. Boothroyd, D. Prabhakaran, and S. W. Lovesey, Science (New York, N.Y.) 332, 696 (2011).

11 C. Dallera, M. Grioni, A. Shukla, G. Vankó, J. Sarrao, J. Rueff, and D. Cox, Physical Review Letters 88, 196403 (2002).

12 N. Jaouen, S. G. Chiuzbian, C. F. Hague, R. Delaunay, C. Baumier, J. Lüning, A. Rogalev, G. Schmerber, and J.-P. Kappler, "X-ray magnetic circular dichroism of $\mathrm{CeFe}_{2}$ by resonant inelastic x-ray scattering," (2010).

13 M. Sikora, A. Juhin, T.-C. Weng, P. Sainctavit, C. Detlefs, F. de Groot, and P. Glatzel, Physical Review Letters 105, 037202 (2010).

${ }^{14}$ H. Yamaoka, I. Jarrige, N. Tsujii, M. Imai, J.-F. Lin, M. Matsunami, R. Eguchi, M. Arita, K. Shimada, H. Namatame, M. Taniguchi, M. Taguchi, Y. Senba, H. Ohashi, 
N. Hiraoka, H. Ishii, and K.-D. Tsuei, Physical Review B 83, 104525 (2011).

15 J. P. Hill, C.-C. Kao, W. Caliebe, M. Matsubara, A. Kotani, J. Peng, and R. Greene, Physical Review Letters 80, 4967 (1998).

${ }^{16}$ P. Abbamonte, C. Burns, E. Isaacs, P. Platzman, L. Miller, S. Cheong, and M. Klein, "Resonant Inelastic X-Ray Scattering from Valence Excitations in Insulating Copper Oxides," (1999).

17 J. Hancock, G. Chabot-Couture, Y. Li, G. Petrakovski, K. Ishii, I. Jarrige, J. Mizuki, T. Devereaux, and M. Greven, Physical Review B 80, 092509 (2009).

18 S. Grenier, J. Hill, V. Kiryukhin, W. Ku, Y.-J. Kim, K. Thomas, S.-W. Cheong, Y. Tokura, Y. Tomioka, D. Casa, and T. Gog, Physical Review Letters 94, 047203 (2005).

19 M. Moretti Sala, V. Bisogni, C. Aruta, G. Balestrino, H. Berger, N. B. Brookes, G. M. de Luca, D. Di Castro, M. Grioni, M. Guarise, P. G. Medaglia, F. Miletto Granozio, M. Minola, P. Perna, M. Radovic, M. Salluzzo, T. Schmitt, K. J. Zhou, L. Braicovich, and G. Ghiringhelli, New Journal of Physics 13, 043026 (2011).

${ }^{20}$ C. Ulrich, L. J. P. Ament, G. Ghiringhelli, L. Braicovich, M. Moretti Sala, N. Pezzotta, T. Schmitt, G. Khaliullin, J. Van Den Brink, H. Roth, T. Lorenz, and B. Keimer, Physical Review Letters 103 (2009), 10.1103/PhysRevLett.103.107205, arXiv:0908.2497.

21 G. Ghiringhelli, A. Piazzalunga, X. Wang, A. Bendounan, H. Berger, F. Bottegoni, N. Christensen, C. Dallera, M. Grioni, J. C. Grivel, M. Moretti Sala, L. Patthey, J. Schlappa, T. Schmitt, V. Strocov, and L. Braicovich, in European Physical Journal: Special Topics, Vol. 169 (2009) pp. 199-205.

22 L. Braicovich, L. J. P. Ament, V. Bisogni, F. Forte, C. Aruta, G. Balestrino, N. B. Brookes, G. M. De Luca, P. G. Medaglia, F. M. Granozio, M. Radovic, M. Salluzzo, J. Van Den Brink, and G. Ghiringhelli, Physical Review Letters 102 (2009), 10.1103/PhysRevLett.102.167401.

${ }^{23}$ L. Braicovich, M. Moretti Sala, L. J. P. Ament, V. Bisogni, M. Minola, G. Balestrino, D. Di Castro, G. M. De Luca, M. Salluzzo, G. Ghiringhelli, and J. Van Den Brink, Physical Review B - Condensed Matter and Materials Physics 81 (2010), 10.1103/PhysRevB.81.174533.

24 M. Guarise, B. Dalla Piazza, M. Moretti Sala, G. Ghiringhelli, L. Braicovich, H. Berger, J. N. Hancock, D. van der Marel, T. Schmitt, V. N. Strocov, L. J. P. Ament, J. van den Brink, P.-H. Lin, P. Xu, H. M. Rø nnow, and M. Grioni, Physical Review Letters 105, 157006 (2010).

25 J. P. Hill, G. Blumberg, Y.-J. Kim, D. S. Ellis, S. Wakimoto, R. J. Birgeneau, S. Komiya, Y. Ando, B. Liang, R. L. Greene, D. Casa, and T. Gog, Physical Review Letters 100, 097001 (2008).

26 D. S. Ellis, J. Kim, J. P. Hill, S. Wakimoto, R. J. Birgeneau, Y. Shvyd'Ko, D. Casa, T. Gog, K. Ishii, K. Ikeuchi, A. Paramekanti, and Y. J. Kim, Physical Review B - Condensed Matter and Materials Physics 81 (2010),
10.1103/PhysRevB.81.085124, arXiv:1002.1440.

27 M. Le Tacon, G. Ghiringhelli, J. Chaloupka, M. M. Sala, V. Hinkov, M. W. Haverkort, M. Minola, M. Bakr, K. J. Zhou, S. Blanco-Canosa, C. Monney, Y. T. Song, G. L. Sun, C. T. Lin, G. M. De Luca, M. Salluzzo, G. Khaliullin, T. Schmitt, L. Braicovich, and B. Keimer, Nature Physics 7, 725 (2011).

28 J. N. Hancock, G. Chabot-Couture, and M. Greven, New Journal of Physics 12 (2010), 10.1088/13672630/12/3/033001, arXiv:1004.0859.

29 M. Z. Hasan, Science 288, 1811 (2000).

30 J. Schlappa, K. Wohlfeld, K. J. Zhou, M. Mourigal, M. W. Haverkort, V. N. Strocov, L. Hozoi, C. Monney, S. Nishimoto, S. Singh, A. Revcolevschi, J.-S. Caux, L. Patthey, H. M. Rø nnow, J. van den Brink, and T. Schmitt, Nature 485, 82 (2012).

31 L. J. P. Ament, G. Ghiringhelli, M. M. Sala, L. Braicovich, and J. Van Den Brink, Physical Review Letters 103 (2009), 10.1103/PhysRevLett.103.117003.

32 J. Kim, D. Casa, M. H. Upton, T. Gog, Y.-J. Kim, J. F. Mitchell, M. van Veenendaal, M. Daghofer, J. van den Brink, G. Khaliullin, and B. J. Kim, Physical Review Letters 108, 177003 (2012).

33 A. C. Hewson, The Kondo Problem to Heavy Fermions (Cambridge University Press, Cambridge, UK, 1993).

34 M. Dzero, K. Sun, V. Galitski, and P. Coleman, Physical Review Letters 104, 106408 (2010).

35 S.-C. Weng, R. Xu, A. H. Said, B. M. Leu, Y. Ding, H. Hong, X. Fang, M. Y. Chou, A. Bosak, P. Abbamonte, S. L. Cooper, E. Fradkin, S.-L. Chang, and T.-C. Chiang, EPL (Europhysics Letters) 107, 36006 (2014).

36 S. Garner, J. Hancock, Y. Rodriguez, Z. Schlesinger, B. Bucher, Z. Fisk, and J. Sarrao, Physical Review B 62, R4778 (2000).

37 J. Hancock, C. Turpen, Z. Schlesinger, G. Kowach, and A. Ramirez, Physical Review Letters 93, 225501 (2004).

38 J. Hancock, T. McKnew, Z. Schlesinger, J. Sarrao, and Z. Fisk, Physical Review B 73, 125119 (2006).

39 J. Sarrao, A. Ramirez, T. Darling, F. Freibert, A. Migliori, C. Immer, Z. Fisk, and Y. Uwatoko, "Thermodynamics of the first-order valence transition in YbInCu4," (1998).

40 J. Sarrao, "Physics of YbInCu4 and related compounds," (1999).

41 J. Hancock, T. McKnew, Z. Schlesinger, J. Sarrao, and Z. Fisk, Physical Review Letters 92, 186405 (2004).

${ }^{42}$ I. Jarrige, A. Kotani, H. Yamaoka, N. Tsujii, K. Ishii, M. Upton, D. Casa, J. Kim, T. Gog, and J. Hancock, Physical Review Letters 114, 126401 (2015).

43 Y. Shvydko, J. Hill, C. Burns, D. Coburn, B. Brajuskovic, D. Casa, K. Goetze, T. Gog, R. Khachatryan, J.-H. Kim, C. Kodituwakku, M. Ramanathan, T. Roberts, A. Said, H. Sinn, D. Shu, S. Stoupin, M. Upton, M. Wieczorek, and H. Yavas, Journal of Electron Spectroscopy and Related Phenomena 188, 140 (2013).

44 H. Okamura, T. Michizawa, T. Nanba, and T. Ebihara, Physical Review B 75, 041101 (2007). 